\title{
Evaluación de la adaptabilidad de abejas nativas sin aguijón en cajas racionales en el corregimiento de Tutunendo, Quibdó, Chocó, Colombia
}

\section{Evaluation of the adaptability of native stingless bees in boxes in the village of Tutunendo, Quibdó, Chocó, Colombia}

\author{
Mirla Perea Murillo*
}

\section{Resumen}

El estudio se llevó a cabo teniendo en cuenta la tendencia actual de desarrollar mecanismos y/o alternativas productivas amigables con el ambiente a partir de la oferta biótica y actividades productivas desarrolladas ancestralmente por las comunidades del corregimiento de Tutunendo, Chocó, Colombia, en relación con el manejo y aprovechamiento de las abejas sin aguijón en cajas racionales. Con el propósito de determinar la adaptación de las especies Tetragonisca angustula y Melipona eburnea en cajas prefabricadas, los volúmenes de productividad y rendimiento de miel, se realizó un análisis de literatura secundaria, se aplicaron métodos tradicionales utilizados por abejeros nativos para aprovechamiento de miel, como la exploración e identificación de nidos en campo teniendo en cuenta las fases de la luna, se utilizó el método técnico invasivo (traslado del medio silvestre aldoméstico), se fabricaron cajas racionales con medidas de $20 \mathrm{~cm}$ de largo por $25 \mathrm{~cm}$ de ancho y $10 \mathrm{~cm}$ de alto y se realizaron monitoreos semanales, partiendo de experiencias desarrolladas a nivel nacional e internacional por apicultores. Todo esto permitió la identificación de las abejas, evaluar la adaptabilidad en cajas racionales, medición de subproductos, volúmenes de producción y manejo de plagas y enfermedades en cajas racionales durante 5 meses. Como resultado se obtuvo que M. eburnea produjo en medio silvestre y en cajas racionales los más altos volúmenes de producción con $9.051 \mathrm{ml}$ de miel, a diferencia de $15 \mathrm{ml}$ que produjo la especie T. angustula. Se evidenció que la mayor amenaza que enfrentan las colmenas son las hormigas arrieras (Atta cephalotes) y la invasión de fóridos (moscas) en cajas racionales.

Palabras clave: Abejas nativas, Adaptabilidad, Cajas racionales, Miel.
* Investigadora Asociada tipo I, Instituto de Investigaciones Ambientales del Pacífico (IIAP), Quibdó, Colombia. e-mail. mperea@iiap.org.co 


\section{Adaptabilidad de abejas nativas sin aguijón. M Perea Murillo}

\section{Abstract}

The study was carried out taking into account the current trend of developing environmentally friendly production mechanisms and/or alternatives based on the biotic supply and productive activities developed ancestrally by the communities of the Tutunendo corregimiento, in relation to the management and use of stingless bees in rational boxes. In order to determine the adaptation of the species (Tetragonisca angustula and Melipona eburnea) in prefabricated boxes, the volumes of productivity and yield of honey, traditional methods used bynative beekeepers for honey exploitation, such as the exploration and identification of nests, were applied. in the field taking into account the phases of the moon; an analysis of secondary literature was made, the invasive technicalmethod was used (transfer from the wild to the domestic), rational boxes were manufactured with measures of $20 \mathrm{~cm}$ long $x 25$ $\mathrm{cm}$ wide and $10 \mathrm{~cm}$ of high and weekly monitoring was carried out, based on developed experiences nationally and internationally by beekeepers. All this, allowed the identification of bees, evaluate the adaptability in rational boxes, measurement of by-products, production volumes and management of pests and diseases in rational boxes for 5 months. As a result, it was obtained that M. eburnea produced in the wild and in rational boxes the highest production volumes with 9.051 $\mathrm{ml}$ of honey, unlike $15 \mathrm{ml}$ produced by the species T. angustula. It was evidenced that the biggest threat facing the hives are the arriera ants (Atta cephalotes) and the invasion of phorids (flies) in rational boxes.

Keywords: Adaptability, Honey, Native bees, Rational boxes.

\section{Introducción}

Las abejas sin agujón son insectos que se caracterizan por presentar diversos patrones de comportamiento, desde especies cleptobiológicas o ladronas, hasta distintos hábitos de nidificación y de recolección de recursos; estas diferencias hacen de cada género y especie particularmente distintos entre sí, siendo estas características las que a su vez marcan la pauta para que su manejo y crianza sea específico dependiendo la especie, en cada sitio donde se aprovechen estas abejas (Reyes 2013).

Las abejas sin aguijón o meliponas son un grupo de insectos sociales que habitan áreas tropicales y subtropicales. A diferencia de la abeja común, originaria del viejo mundo (África), las meliponas son nativas del continente americano donde se han identificado más de 350 especies (Baquero y Stamatti 2007). Se considera uno de los grupos más comunes de insectos, de gran importancia ecológica y económica gracias a sus hábitos alimenticios. La visita a las flores en busca de néctar y polen tiene como consecuencia la polinización de un gran número de plantas de interés para otros organismos (Nates-Parra et al. 2005).

Es importante tener en cuenta que poco se conoce acerca de la estructura y dinámica de las comunidades de abejas sin aguijón y menos aún, acerca de los efectos de las modificaciones en los paisajes naturales sobre estas comunidades, porque la estructura de las comunidades de abejas sin aguijón puede verse afectada por factores tales como la disponibilidad y la competencia por alimento, sitios de nidificación y depredación (Nates-Parra et al. 2008).

En este sentido, en el mundo se conocen aproximadamente 17.000 especies de abejas. El Neotrópico cuenta con casi 6.000 especies, 3.000 de lengua larga (Apidae y Megachilidae) y 3.000 de lengua corta (Colletidae, Andrenidae, Halictidae) (Roubik 1995). Para Colombia se estiman aproximadamente 1.000 especies de abejas, agrupadas en 90 géneros y cinco familias (Roubik 1995). Sin embargo, estudios como el de Smith-Pardo (2003), argumentan que en Colombia 


\section{Bioetnia Volumen 14, 2017}

se encuentran reportadas 466 especies pertenecientes a cinco de las siete familias existentes a nivel mundial, pero estos estudios han sido poco documentados (Nates-Parra et al. 2005); otros estudios más recientes estiman un número mayor a las 800 especies para el país (Vélez 2011).

En los últimos años, Colombia ha empezado a valorar la cría y manejo de abejas sin aguijón; su potencial en nuestro país aún no se ha desarrollado en la misma magnitud que en otros países. Estas producen pequeñas pero importantes cantidades de miel. El conocimiento que actualmente existe en Colombia sobre las abejas silvestres se enfoca especialmente hacia estudios taxonómicos y de historia natural para unos 10 géneros, dentro de los que se destacan las abejas sin aguijón. Este conocimiento ha permitido generar programas dirigidos a establecer técnicas de cría y manejo con fines productivos (Ministerio de Agricultura y Desarrollo Rural 2011).

En Colombia hay aproximadamente 100 especies de esta subfamilia y tan solo 17 son utilizadas para producción de miel, cera, polen o resinas; de ellas, solo 11 se explotan para obtención de miel, ya sea en forma rústica o semidomesticada. Las abejas del género Melipona (M. favosa, M.gr.fasciata, M. interrupta, M. compressipes) y sobre todo Trigona (Tetragonisca angustula) con frecuencia son objeto de búsqueda para obtención de miel de excelente calidad y a la cual la medicina popular le asigna propiedades terapéuticas (Nates-Parra et al. 2005).

En este contexto, la actividad apícola relacionada con la producción de miel en el Chocó Biogeográfico corresponde a un sector emergente, con un buen potencial de mercado, pero que presenta bajos niveles de producción donde la poca credibilidad en el negocio y el bajo conocimiento en el desarrollo del mismo hacen que se invisibilice esta actividad de producción. A pesar de tener una escasa política pública específica, existe interés por quienes realizan esta actividad y los pequeños gremios vienen haciendo un es- fuerzo por consolidarse; no obstante, el sector aún presenta brechas a superar en diversos sectores como la cadena productiva que no ha favorecido su desarrollo en la región y en el país.

Sin embargo, en el departamento del Chocó, el Instituto de Investigaciones Ambientales del Pacífico (IIAP) ha venido desarrollado algunas investigaciones con el fin de crear alternativas sostenibles de producción en el marco de la identificación de abejas nativas, caracterización, flora apícola asociada, evaluación de productos, productividad y conformación de red apícola en las estaciones ambientales de la institución.

Esta investigación se realizó con el interés de evaluar la adaptabilidad de dos especies de abejas nativas identificadas, trasladadas a cajas racionales previamente construidas y evaluar los volúmenes de miel como estrategia de agronegocios para las familias productoras en las comunidades. De igual manera, se pretende aportar insumos para desarrollar productos competitivos teniendo en cuenta los encadenamientos productivos que se puedan posesionar en nichos de mercado verdes que permita dar el paso al desarrollo de planes de manejo para la conservación sin poner en riesgo la especie.

\section{Materiales y métodos}

Área de estudio. El corregimiento de Tutunendo está localizado a $05^{\circ} 44^{\prime} 41.7^{\prime \prime}$ norte $76^{\circ} 32^{\prime} 24.2^{\prime \prime}$ oeste. Dista 40 minutos del municipio de Quibdó y se destaca por tener un bosque húmedo Tropical (bh-T), fisiográficamente ubicado en la zona denominada Selva Pluvial Central (Holdridge 1996, citado por Mejía y Pino 2010). Está ubicado a $150 \mathrm{~m}$ de elevación, con temperatura megatermal $\left(27^{\circ} \mathrm{C}\right)$ y una precipitación promedio anual de $11.700 \mathrm{~mm} / \mathrm{año} \mathrm{(Poveda} \mathrm{et} \mathrm{al.} \mathrm{2004,} \mathrm{citado} \mathrm{por}$ Ramírez 2011). La zona presenta una topografía quebrada y con numerosos cursos de agua; los suelos son ácidos y de fertilidad moderada. El área está cubierta de bosque en diferentes estados 


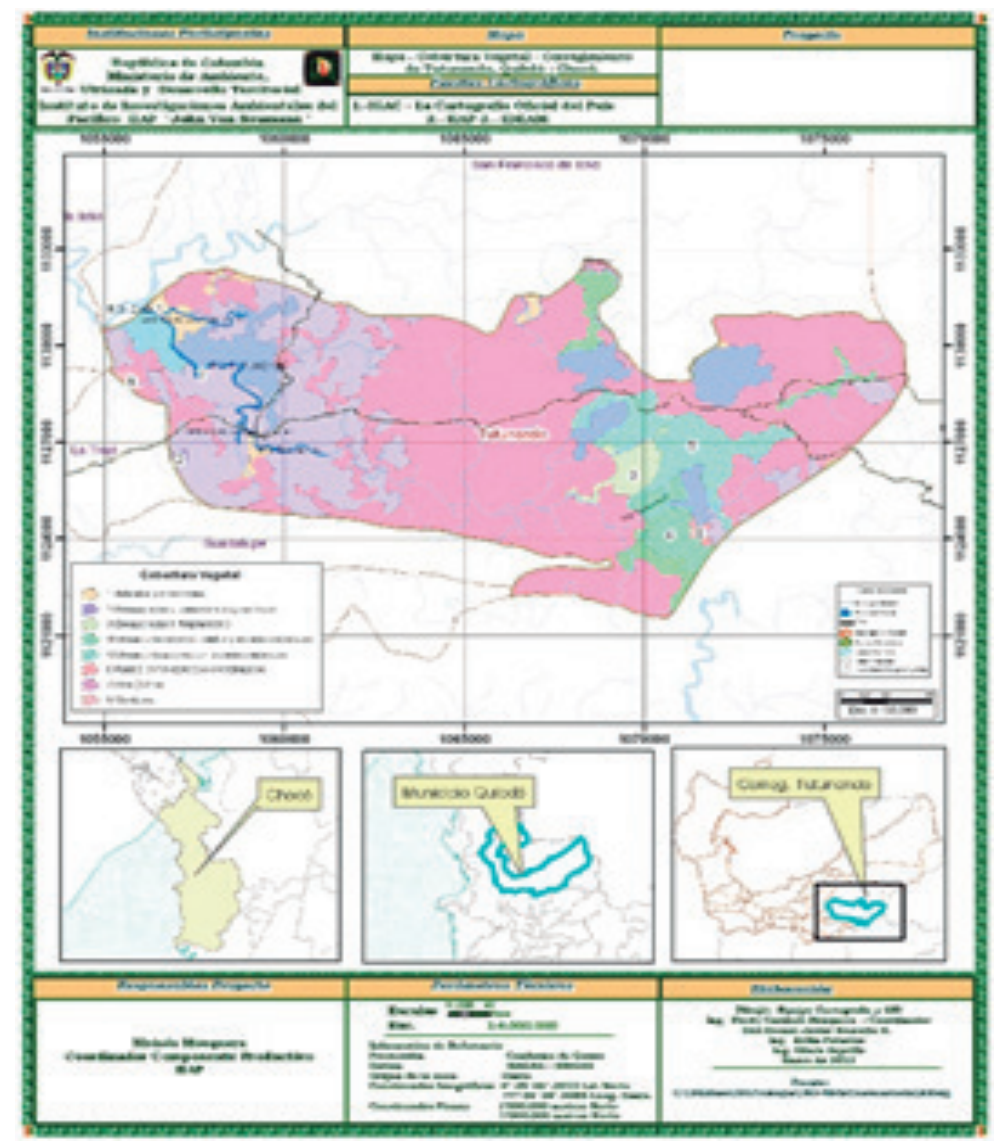

Figura 1. Localización corregimiento de Tutunendo. Cobertura vegetal.

de intervención (Figura 1).

Método. La investigación se desarrolló teniendo en cuenta 2 etapas:

Análisis de literatura secundaria. Esta etapa fue desarrollada teniendo como base trabajos de investigación de fuentes secundarias como publicaciones, documentos y artículos de sitios web relacionados con la identificación de abejas nativas, adaptación de las diferentes especies en cajas racionales, aprovechamiento de subproductos y comercialización de estos, bajo criterios de sostenibilidad ambiental.

Aplicación del método cualitativo-descriptivo. El segundo método utilizado se enmarcó desde un enfoque cualitativo teniendo en cuenta el conocimiento ancestral, la participación directa de los apicultores en la actividad productiva y su interacción con el ambiente natural, donde además se tuvo en cuenta las fases de la luna considerando el contexto físico-espacial, productivo y sociocultural. De igual manera, esta etapa permitió la ejecución de la investigación desarrollando las siguientes actividades:

Identificación de abejas nativas: Se realizaron ocho salidas exploratorias en áreas boscosas aledañas al corregimiento de Tutunendo con el fin de conocer la localización y características de nidos silvestres. La búsqueda fue principalmente hasta donde la vista les permitía distinguir los tubos de ingreso y las abejas sobrevolando el mismo; en algunos casos se ubicaron nidos por el zumbido revelador en caminatas. Una vez ubicadas e identificadas las colmenas se procedió a identificar el tipo, especie, nombre vulgar, descripción general a nivel de estructura y composición de cada colmena identificada, para su posterior seguimiento y 


\section{Bioetnia Volumen 14, 2017}

determinación de los volúmenes de productividad a nivel de miel. Para esta actividad se requirió del acompañamiento de apicultores empíricos reconocidos por la comunidad, donde luego de ubicados los nidos y colmenas, se colectaron especímenes y se llevaron al Laboratorio de Limnología de la Universidad Tecnológica del Chocó (UTCH), donde, a través de claves taxonómicas, identificaron las especies encontradas.

Construcción e instalación de cajas racionales: Se construyeron nueve cajas racionales utilizando madera desechada. Las cajas presentaban una estructura compuesta por tres gavetas removibles para el almacenamiento de miel y una cubierta; cada gaveta medía $20 \mathrm{~cm}$ de largo por $25 \mathrm{~cm}$ de ancho y $10 \mathrm{~cm}$ de alto. En su interior, la segunda gaveta estaba compuesta por una cámara triangular que facilitaba la cosecha de la miel y un orificio que permitía la entrada y salida de las abejas.

Para la instalación de las mismas se tuvo en cuenta que los sitios dispuestos por los apicultores estuvieran bajo sombríos cerca de fuentes proteínicas como flores y fuentes de agua, como también, que las cajas racionales no estuvieran expuestas a fuertes vientos y a depredadores rastreros.

Traslado de abejas nativas a cajas racionales: Se utilizó el método invasivo, el cual consiste en el traslado del nido desde su habitad natural hasta las cajas racionales. Esta actividad se desarrolló en días poco soleados, para evitar la menor perturbación posible a la colmena.

Una vez localizados los nidos, se procedió a la extracción de las colmenas. En dos de los casos, se requirió realizar varios orificios en árboles para levantar la corteza y extraer la colonia; en cinco casos, se desenterró la colonia localizada bajo rocas y piedras, y en dos se levantaron ladrillos para extraer las colonias incrustadas en cemento de casas en estado de abandono.

El traslado de las colonias a las cajas racionales se realizó teniendo en cuenta la metodología descrita por Miembros de la Asociación de Productores de Yateí de Misiones y CEDIT (2005) en la cual se tienen en cuenta los siguientes aspectos:

- Localizar la o las celdas reales que se encuentren en la colonia.

- Transferir los discos reales a las cajas racionales evitando el menor estrés posible.

- Ubicar los discos de la colmena en la misma posición encontrada.

- Aislar la colmena del suelo para evitar entrada de depredadores.

- Cubrir la colmena con láminas de zinc para evitar la humedad por las altas precipitaciones. Monitoreo de las colonias en cajas modulares: Luego de trasladadas las colonias a las cajas racionales, se hizo el seguimiento y monitoreo realizado una vez por semana (entre las $10 \mathrm{am}$ y 2 pm); esta actividad de observación permitió determinar el estado de la estructura de la colmena, estado fitosanitario, y presencia o ausencia de plagas y/o enfermedades.

Medición de subproductos y evaluación de volúmenes de producción: Para el seguimiento de las colmenas silvestres y domesticadas, se elaboró la ficha de seguimiento que aparece en la Tabla 1.

Esta etapa de la investigación se realizó a través

Tabla 1. Formato para el monitoreo de colmenas

\begin{tabular}{|c|c|c|c|c|c|c|}
\hline \multirow[t]{2}{*}{ Colmena $\mathrm{N}^{\circ}$} & \multirow[t]{2}{*}{ Coordenadas } & \multirow[t]{2}{*}{ Localidad } & \multirow{2}{*}{$\begin{array}{c}\text { Especie de } \\
\text { abeja }\end{array}$} & \multirow{2}{*}{$\begin{array}{c}\text { Plagas y } \\
\text { enfermedades }\end{array}$} & Volumen de & \multirow{2}{*}{$\begin{array}{l}\text { Informante } \\
\text { clave }\end{array}$} \\
\hline & & & & & 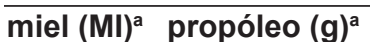 & \\
\hline & & & & & & \\
\hline & & & & & & \\
\hline & & & & & & \\
\hline & & & & & & \\
\hline
\end{tabular}


de la medición de las cantidades de los subproductos (miel) en un período de cinco meses; las mediciones fueron realizadas utilizando equipos y utensilios estandarizados como bureta calibrada a escala mililitros y jeringa plástica. Para la extracción de la miel en cajas racionales, se utilizó una jeringa plástica de $10 \mathrm{~cm}$ con la cual se extraía la miel como producto principal; su extracción se realizó con precaución con el objeto de ocasionar la menor perturbación posible a los individuos presentes en las colmenas. Estas mediciones se realizaron teniendo en cuenta aspectos que permitieron la evaluación como las fases lunares, el tipo de especie, características organolépticas de la miel y volúmenes de producción.

No existen normas de higiene, ni utensilios que garanticen la inocuidad y la calidad de la miel "proceso trasmitido de generación en generación" (Universidad Nacional 2015). Así entonces, la evaluación de los subproductos se dio a partir de los seguimientos y monitoreos, luego del traslado de nidos naturales a cajas modulares. Las colmenas se dejaron durante un período de dos meses sin tocar para que se fortalecieran en su forma de anidación, estructura y adaptación.

Cálculo estadístico. Se utilizó la prueba del análisis de varianza a través del programa estadístico $\mathrm{R}$, con el fin de hacer inferencias sobre los factores y las variables dependientes; se contrastó la hipótesis de las medias y se aplicó el test de normalidad Shapiro-Wilk para determinar la normalidad de una muestra aleatoria.

Manejo de plagas y enfermedades. Para el manejo de plagas y enfermedades se recurrió al monitoreo semanal y apoyados en la observación detallada de cada una de las cajas racionales se determinaba si había presencia de algún organismo externo que pudiera hacerles daño a las colmenas.

\section{Resultados y discusión}

Identificación de abejas nativas. De acuerdo con las exploraciones realizadas en compañía de apicultores nativos del corregimiento de Tutunendo y teniendo en cuenta las características conductuales, morfológicas y estructurales de anidación, se localizaron nueve nidos con sus respectivas colmenas de abejas nativas sin agujón, de los cuales cinco correspondían a la especie Melipona eburnea y cuatro a la especie Tetragonisca angustula (Figura 2), según la clasificación taxonómica determinada por el Laboratorio de Entomología de la UTCH, las cuales se detallan a continuación:

Melipona eburnea

Filo: Arthrópoda

Clase: Hexápoda

Orden: Hymenóptera

Suborden: Aculeata

Familia: Apideae
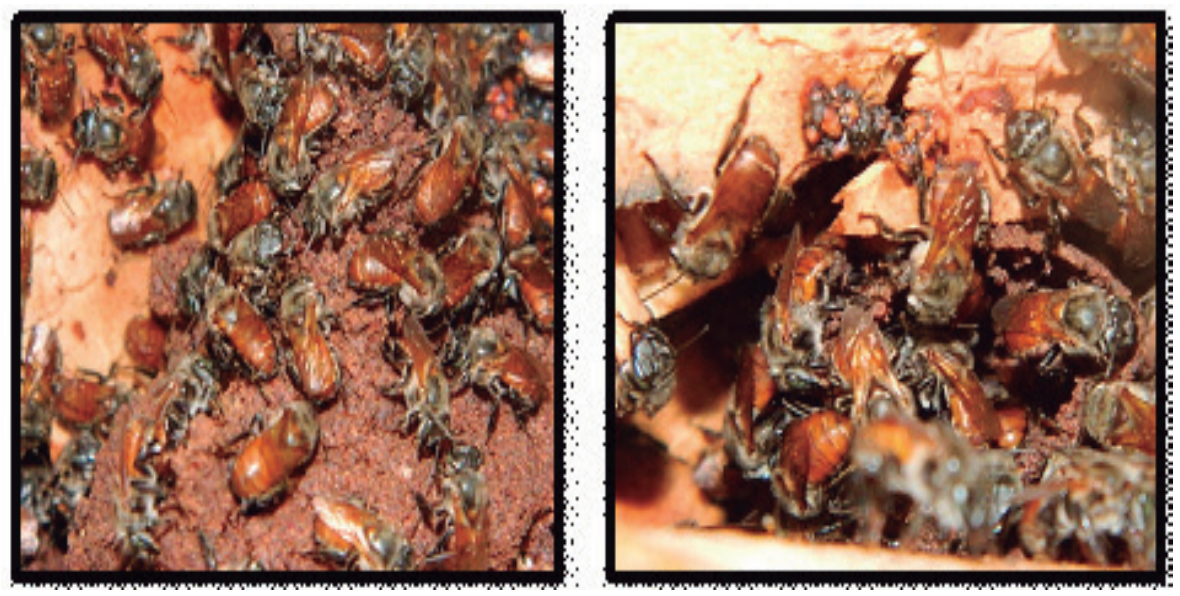

Figura 2. Melipona eburnea. 


\section{Bioetnia Volumen 14, 2017}
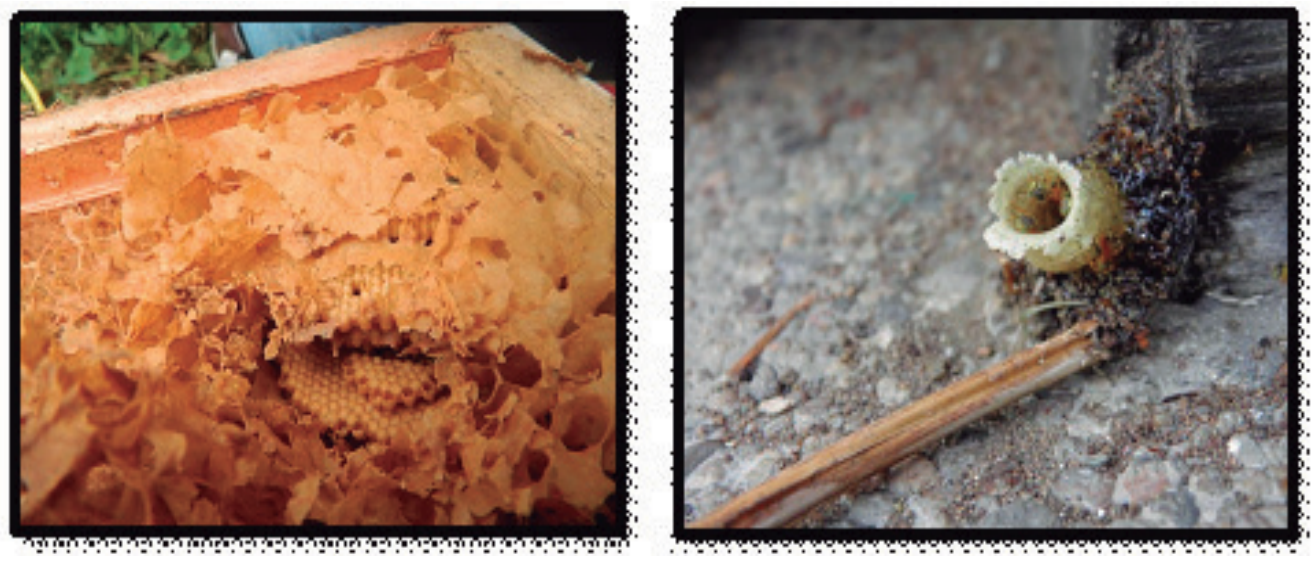

Figura 3. Tetragonisca angustula.

Género: Melipona

Especie: Melipona eburnea

Es una abeja predominantemente amazónica encontrada en Colombia, Perú, Bolivia y Brasil. En Colombia se encuentra en la Amazonia, región andina y Orinoquia. Habita entre los 800-2.100 msnm. Es de tamaño grande, generalmente de color café oscuras en la parte dorsal y color negro en la cabeza, patas y abdomen. Se caracterizan por tener un penacho de pelos rojizos en los ángulos laterales superiores del escuto y marcas claras en la cara (Otero 2017). Face negra, excepto por una banda media poco distinguible sobre el clipeo; tergitos basales poco peludos; tergo 1 casi completamente amarillo pálido; tergos 4-5 cubiertos con pelos plumosos (Figura 3). Porción lateral de tergo 5 con pelos erectos, largos, negros o marrón oscuro, con apariencia espinosa (Amat et al. 2007).

Tetragonisca aungustula

Filo: Arthrópoda

Clase: Insecta

Orden: Hymenóptera

Suborden: Apocrita

Familia: Apideae

Género: Trigona Roubik DW. 1995. Polinización de plantas cultivadas en los trópicos.

Especie: Tetragonisca angustula

Es una de las abejas sin aguijón más comunes. Se distribuye desde el sur de México al norte de Argentina. Según Schwarz y Bacon (1948) y
Roubik (1995) esta abeja se caracteriza por su apariencia delgada, frágil y tamaño pequeño, aproximadamente $4 \mathrm{~mm}$ de longitud; ancho máximo de la cabeza: $1.8 \mathrm{~mm}$. Cuerpo predominantemente de color naranja, sobre todo el metasoma y patas; alas ennegrecidas y partes de la cara y antenas amarillas. Tibias posteriores claviformes y con una pequeña corbícula limitada al quinto apical de la tibia y no ocupando todo el ancho de la misma.

Hacen nidos cubiertos en casi cualquier cavidad que encuentren disponible. La entrada al nido construido con cerumen es un tubo de cera de color claro, porosa, generalmente impregnado de resinas, para defenderse de ocasionales invasores. Son muy apreciadas por la calidad de su miel.

Las características paisajísticas fue un factor determinante en la descripción de los nidos, identificación de las colonias y las especies de abejas encontradas de acuerdo con Nates-Parra y Rodríguez (2011) quien comenta que los ciclos de floración, la disponibilidad, forma y distribución de los recursos también son factores determinantes en la actividad de forrajeo y desarrollo de las colmenas.

Por otra parte, fue muy importante tener en cuenta el sustrato de anidación de las especies encontradas; para el caso de M. eburnea, localizada en dos árboles vivos en pie, correspondiente a las especies vegetales boteco (Cuararibea sp.) y trúntago (Vitex columbiensis) de interés especial 
para las comunidades. Teniendo en cuenta las familias a las que pertenece cada árbol, las cajas racionales se ubicaron en área donde hubiese plantas de la familia Malvaceae para el primero y Verbenaceae para el segundo. Es importante recalcar que para estas dos colonias funcionó la estrategia de ubicar las cajas racionales cerca a estas dos familias de plantas, pues fueron las más sobresalientes a nivel de producción de miel; sin embargo, estudios como el de Miembros de la Asociación de Productores de Yateí de Misiones y CEDIT 2005), argumentan que por no haber estudios suficientes que demuestren cuáles son las plantas que dependen efectivamente de la visita de las abejas nativas, debilita un poco los procesos de producción de miel en cajas racionales, porque se pudieran tener mejor resultados en la domesticación de las abejas al tener identificadas las especies vegetales más visitadas.

Para el caso de los cinco nidos encontrados debajo de la tierra y de rocas, cuatro de estos correspondía a M. eburnea y uno a T. angustula quienes se encontraban cerca de especies vegetales tales como aserrín (Penthacletra macroloba), aliso (Piptocoma discolor), galán de noche (Cestrum nocturnum), mora silvestre (Rubus froribundus), pipi longo (Piper aduncum) y algodoncillo (Luehea candida) de donde obtenían su alimento. La presencia de estas especies vegetales cerca de los nidos indujo al equipo investigativo a ubicar las

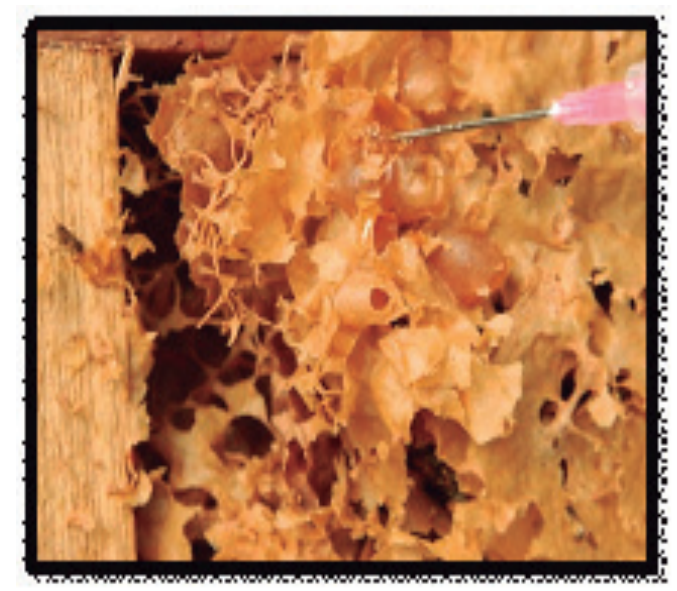

cajas racionales cerca de las mismas especies o que pertenecieran a la misma familia de alguna de ellas, tal es el caso de la Santa María perteneciente a la familia Piperaceae al igual que el Piper aduncum y el lulo (Solanum quitoense).

Evaluación de volúmenes de producción. La construcción de recipientes para almacenamiento de alimento se detectó en el primer monitoreo realizado dos meses después de instaladas las colmenas. Se observó el involucro de la cría totalmente sellado. El número de recipientes de miel y polen en el área de almacenamiento fue incontable, debido al orden de construcción de las colmenas (Figura 4). De igual manera, el monitoreo permitió evaluar la productividad y la medición de cantidades y calidades de productos elaborados por las abejas si aguijón luego de cinco meses.

Así entonces, el seguimiento permitió determinar que la especie M. eburnea se considera como la especie más representativa en el proceso investigativo por sus características particulares de adaptación en cajas racionales que presentó los más altos niveles de producción con un total de $9.051 \mathrm{ml}$ de miel a diferencia de la especie T. angustula que fue de $15 \mathrm{ml}$ de miel. Este resultado fue corroborado por los apicultores que hicieron el acompañamiento en la investigación, pues argumentan que la especie $M$. eburnea se considera como una de las abejas nativas más

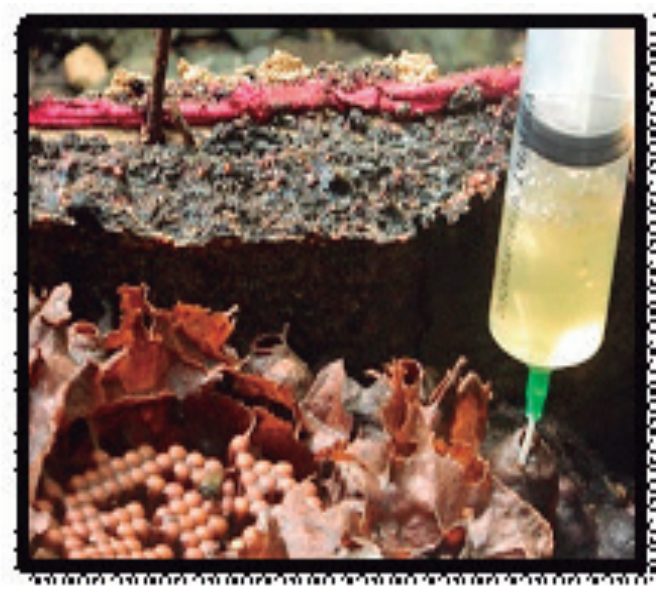

Figura 4. Extracción de miel almacenada. 


\section{Bioetnia Volumen 14, 2017}

productoras de miel, por ello es tan apetecida por apicultores y aserradores, amenazando su existencia. Sin embargo, aunque $M$. eburnea arrojó esos resultados, es importante anotar que la $T$. angustula produce menos miel, pero las características organolépticas y medicinales la hacen más apetecida en el mercado y por lo tanto su valor económico es mayor.

Esta diferencia significativa, se debe a que según Nates-Parra et al. (2005), la especie T. angustula es más vulnerable al ataque de plagas como se evidenció en la investigación en cuatro de las cajas racionales establecidas, lo cual afectó la producción. Se puede decir que la adaptación de la M. eburnea a las cajas racionales, es una característica propia de la especie, pues estas abejas se caracterizan por presentar colmenas muy grandes, con gran cantidad de individuos y resistentes a vientos fuertes y precipitaciones.

De igual manera, la producción de miel por colmena es muy variable debido a las condiciones ambientales paisajísticas, incluso de la misma zona. En promedio, pueden esperarse producciones (en litros por colonia al año) entre uno y cuatro litros para especies del género Melipona; entre $500 \mathrm{ml}$ y un litro para T. angustula, y entre 0,4 y 8 litros para otras especies (Ascencio 2014)

Otro aspecto relevante de las dos especies estudiadas es que son endémicas y cada una se alimenta de un tipo de vegetación diferente. Se mueven en rangos de altutud de 0-500 m, de $500-1.500 \mathrm{~m}$ y por encima de $1.500 \mathrm{~m}$ según la Universidad Nacional (2015); la T. angustula en particular es generalista, se adapta fácilmente a cualquier región (selvas, zonas secas y ciudades) a diferencia de la M. eburnea, que se destaca por encontrarse solo en zonas selváticas.

Cálculo estadístico. A través del programa estadístico R y de acuerdo con la prueba utilizada, análisis de varianza, se evidenció diferencias estadísticamente significativas de la producción de miel; esto se pudo comprobar aplicando el test de normalidad Shapiro-Wilk (Figura 5) que muestra que solo uno de los datos (el primero) se encuentra disperso dentro del rango 0-1.000 $\mathrm{ml}$, lo cual indica que es el rango de distribución de los volúmenes de miel evaluados; además, la distribución de los datos muestra una mediana de $500 \mathrm{ml}$ que corresponde al 50\%; sin embargo, la mayor distribución de los datos se encuentran en el cuartil tres de la caja.

Con respecto a los muestreos establecidos en este estudio y las dos especies de abejas nativas analizadas, los niveles de producción de la especie M. eburnea superaron en un $100 \%$ a la especie T. angustula, lo cual fue manifestado por

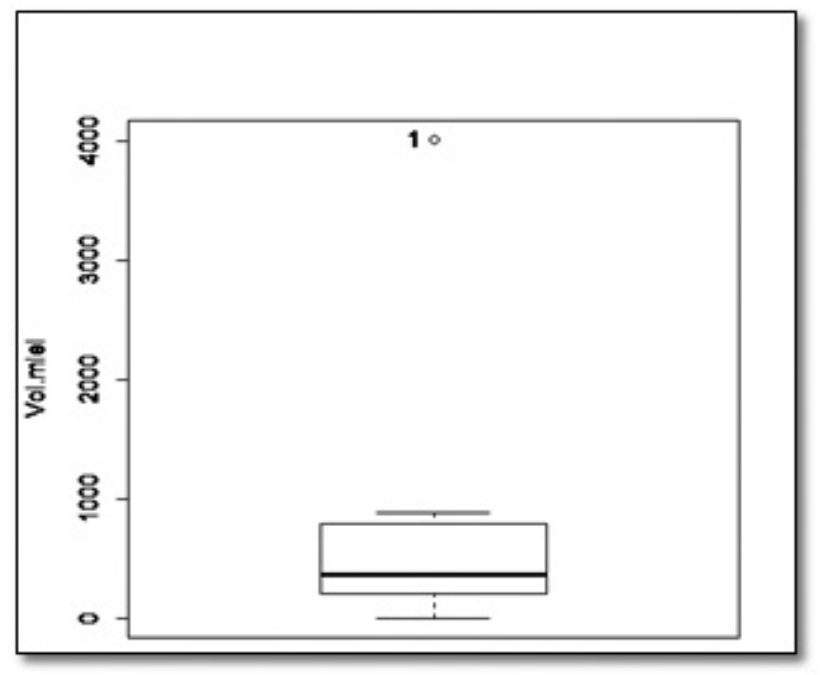

Figura 5. Caja de bigotes análisis de volúmenes de miel de las especies M. eburnea y T. angustula. 
los apicultores, quienes practican esta actividad ancestralmente y que además argumentaban en las entrevistas informales antes y durante el desarrollo de la investigación, los altos niveles de producción de $M$. eburnea.

Manejo de plagas y enfermedades. Luego de dos meses de constantes monitoreos, las colmenas se encontraron bien fortalecidas y en condiciones óptimas para la producción, sin embargo, los resultados del traslado no fueron positivos debido a la presencia de plagas como fóridos y hormigas que atacaron cuatro de las cajas racionales establecidas; esto se debe a que probablemente los apicultores no realizaron los monitoreos periódicos día a día, lo cual hubiera podido evitar el ataque de estas plagas.

\section{Conclusiones}

Esta investigación hace una aproximación a la diversidad asociada con la meliponicultura para el Chocó Biogeográfico, pero se reconoce que existen grandes falencias en lo que respecta al conocimiento de esa diversidad y su manejo; además el interés biológico por este grupo, los ingresos generados por el comercio de miel, cera y otros productos, han impulsado la búsqueda de información local sobre biología y manejo racional de estas especies en el rango de su distribución.

Por otro lado, el escaso trabajo de colecta y la falta de taxónomos especialistas limitan el conocimiento de las abejas sin aguijón y reduce las posibilidades del uso de sus productos directos e indirectos. El presente proyecto se desarrolló teniendo en cuenta la colecta sistemática para el grupo de los meliponinos realizado en el departamento del Chocó; así también, este tipo de investigación se destaca porque permitirá conocer la riqueza biológica de este grupo de insectos y con base en éste será posible desarrollar trabajos productivos enfocados en la polinización de cultivos de forma más eficientes y encontrar especies de meliponinos que pueden ser usadas por las comunidades humanas para obtener productos como la miel, propóleo y cera. Esto puede redundar en un beneficio económico adicional para la población.

En este sentido, resulta una costumbre hasta la actualidad ver a las abejas sin aguijón como símbolo cultural y esto hace que los pequeños productores se apeguen al sistema tradicional o uso de los troncos huecos. Sin embargo, los apicultores bajo comprobación y muestra de los resultados obtenidos, están dispuesto a trabajar con cajas racionales y teniendo en cuenta los costos de producción, hasta el momento no existe información precisa para determinarlos e incurrir el apicultor para llevar a cabo sus procesos productivos.

La explotación regional por parte de los “cazadores de miel" ha diezmado algunas poblaciones en algunas regiones del país. Las abejas del género Melipona en particular son muy sensibles a perturbaciones de su hábitat, de ahí el caso de M. eburnea, quien se encuentra en categoría de amenaza debido a la presión que se ejerce sobre ella y que al pasar el tiempo y poder encontrar nidos de esta especie se debe adentrar mucho en el bosque; por otro lado, el establecimiento de asentamientos humanos en áreas antes boscosas ha contribuido a su disminución y la deforestación generalizada ha reducido sus sitios de nidificación.

\section{Literatura citada}

Amat GD, Amat E, Andrade G. 2007. Libro rojo de los invertebrados terrestres de Colombia. Bogotá: Conservación Internacional, Universidad Nacional. Disponible en: https://www.researchgate.net/publication/267333768 Libro Rojo de los Invertebrados terrestres de Colombia_Contenido

Ascencio DJ. 2014. Evaluación de los cambios pre y postcosecha de la miel de especies de abejas sin aguijón. (Tesis de grado). Bogotá: Universidad Nacional de Colombia, Facultad de Ciencias Maestría en Ciencia y Tecnología de Alimentos; 208 pp. Disponible en: http:// www.bdigital.unal.edu.co/46598/1/1107551.2014.pdf Baquero GL, Stamatti G. 2007. Cría y manejo de abejas 


\section{Bioetnia Volumen 14, 2017}

sin aguijón. Tucumán: Ediciones del subtrópico. Disponible en: https://issuu.com/abejassilvestres2013/ docs/cria y manejo de abejas sin aguijon argentina 2007

Mejía HJ, Pino N. 2010. Diversidad de orquídeas epífitas en un bosque húmedo tropical (bh-T) del departamento del Chocó, Colombia. Acta Biol Colomb. 15 (2): 37-46. Disponible en: https://webcache.googleusercontent. com/search?q=cache:GaHMY-PV4gIJ:https://revistas. unal.edu.co/index.php/actabiol/article/view/9377+\&c$\underline{\mathrm{d}=1 \& \mathrm{hl}=\mathrm{es} \& \mathrm{ct}=\mathrm{clnk} \& \mathrm{gl}=\mathrm{co}}$

Miembros de la Asociación de Productores de Yateí de Misiones y CEDIT. 2005. Características y características de las yatei y otras meliponas. Disponible en: https:// issuu.com/marcoacuna/docs/meliponas yatei tetragonisca angust

Ministerio de Agricultura y Desarrollo Rural. 2011. Diagnóstico de la actividad apícola y de la crianza de las abejas en Colombia. MinAgricultura, IICA. Bogotá: IICA. Disponible en: http://conectarural.org/sitio/ sites/default/files/documentos/diagnostico-de-la-actividad-apicola-y-de-la-crianza-de-abejas-en-colombia. pdf

Nates-Parra G. 2005. Abejas silvestres y polinización. Manejo Integrado de Plagas y Agroecología. 75: 7-20. Disponible en: http://orton.catie.ac.cr/repdoc/A1865e/ A1865e.pdf

Nates-Parra G, Palacios E, Parra A. 2008. Efecto del cambio del paisaje en la estructura de la comunidad de abejas sin aguijón(Himenoptera:Apidae) en Meta, Colombia. Rev Biol Trop. 56 (3): 1-11. Disponible en: https://revistas. ucr.ac.cr/index.php/rbt/article/view/5711

Nates-Parra G, Rodríguez A. 2011. Forrajeo de colonias de Melipona eburnea (Himenóptera: Apide) en el piedemonte llanero, Meta, Colombia. Rev Colomb Entomol. 37(1): 121-7.Disponible en: http://www.scielo.org.co/ pdf/rcen/v37n1/v37n1a22.pdf
Otero G. 2017. Meliponas de Colombia. Disponible en: https://www.meliponasdecolombia.com

Ramírez GG, Galeano G. 2011. Comunidades de palmas en dos bosques del Chocó Colombia. Caldasia.33 (2):31529. Disponible en: https://webcache.googleusercontent. com/search?q=cache:bYXz6dnk608J:https://revistas. unal.edu.co/index.php/cal/article/view/36393+\&c$\mathrm{d}=1 \& \mathrm{hl}=\mathrm{es} \& \mathrm{ct}=\mathrm{clnk} \& \mathrm{gl}=\mathrm{co}$

Reyes A. 2013. Manejo y conservación de abejas sin aguijón (Apidae: Meliponini) en una región de balsas Michoacano, México. VIII Congreso Mesoamericano de Abejas Nativas, Heredia, Costa Rica. Disponible en: https:// www.researchgate.net/publication/273124254 MANEJO Y CONSERVACION DE ABEJAS SIN AGUIJON APIDAE MELIPONINI EN UNA REGION DEL BALSAS MICHOACANO MEXICO

Roubik DW. 1995. Polinización de plantas cultivadas en los trópicos. Boletín de Servicios Agrícolas de la FAO. 118: 1-6.

Schwarz HF, Bacon AL. 1948. Stingless bees (Meliponidae) of the Western Hemisphere: Lestrimelitta and the following subgenera of Trigona: Trigona, Paratrigona, Schwarziana, Parapartamona, Cephalotrigona, Oxytrigona, Scaura, and Mourella. Bull AMNH. 90: 1-546.

Smith-Pardo A. 2003. Un preliminar. Cuenta de las abejas en Colombia (Hymenoptera: Apoidea). Presente conocimiento y direcciones futuras. Kansas: Sociedad Entomológica de Kansas.

Universidad Nacional. 2015. Programa estratégico en alternativas para la generación de valor de productos apícolas en Colombia a través de la innovación y el desafio tecnológico. Bogotá: Universidad Nacional.

Vélez RI. 2011. Recuento sobre las publicaciones de abejas silvestres en Colombia. Boletín del Museo Entomológico Francisco Luis Gallego. 3 (3): 15-29. Disponible en: http://ciencias.medellin.unal.edu.co/ museos/entomologico/images/Boletin/2011-09/4.pdf 